\title{
Desempenho de equações bioclimáticas para estimativa da evapotranspiração de referência em Conceição do Araguaia/PA
}

\author{
Elton Ferreira Lima \\ Mestrando em Engenharia Agrícola - Universidade Federal de Santa Maria- UFSM \\ 凶aurelio-mcp@hotmail.com \\ Jhonata Santos Santana \\ Engenheiro Agrônomo, Bolsista de Apoio Técnico Institucional II \\ Universidade Estadual da Região Tocantina do Maranhão- UEMASUL \\ Rafael Guimarães Silva Moraes \\ Mestrando em Produção Vegetal - Universidade Estadual do Norte Fluminense- UENF
}

Bryann Lynconn Araujo Silva Fonseca

Bolsista de Iniciação Científica- Universidade Estadual da Região Tocantina do Maranhão- UEMASUL

Pedro Luid de Sousa Oliveira

Discente de Engenharia Agronômica- Universidade Estadual da Região Tocantina do Maranhão- UEMASUL

\section{Cristiane Matos da Silva}

Mestre em Engenharia de Barragem e Gestão Ambiental

Professora Auxiliar Classe II, no Centro de Ciências Agrárias

Universidade Estadual da Região Tocantina do Maranhão- UEMASUL

Recebido em 21 de maio de 2019

Aceito em 26 de junho de 2019

\section{Resumo:}

Devido às incertezas nos totais pluviométricos que incidem sobre uma região por causa das alterações climáticas, a agricultura precisa dispor de técnicas que minimizem os riscos inerentes dessa atividade. A ETo originalmente introduzida sob o termo evapotranspiração potencial (ETp), é um importante parâmetro agrometeorológico, principalmente para planejamento e manejo de irrigação. Existem diversas equações para calcular a ETo, desde aqueles mais simples, com base puramente empírica, até aquelas que apresentam embasamento físico consistente sobre o fenômeno, contudo, há sempre algum empirismo presente. Diante disso, o presente trabalho teve como objetivo avaliar o desempenho de quatro métodos (Turc, Hargreaves e Samani, Jesen-Haise e Makkink) para estimativa da ETo diária por meio de comparações com o método padrão de Penman-Monteith-FAO56, em Conceição do Araguaia/PA. Os dados meteorológicos foram retirados do Instituto Nacional de Meteorologia no período de 26 anos (1990-2016). O método tomado como padrão foi o de Penman-Monteith-FAO56, e a comparação dos resultados foi por meio do coeficiente de determinação $\left(\mathrm{R}^{2}\right)$, índice de concordância de Willmott $(\mathrm{d})$, coeficiente de correlação de Pearson ( $r$ ) e do coeficiente de confiança (c). Observou-se que nas condições climáticas de Conceição do Araguaia/PA, os métodos de Turc e Hargreavis e Samani foram os que melhor estimaram a evapotranspiração de referência (ETo) quando comparado com o padrão Penman-Monteith-FAO.

Palavras-chave: Agrometeorologia, evaporação, irrigação, Penman-Monteith. 
Desempenho de equações bioclimáticas para estimativa da

evapotranspiração de referência em Conceição do Araguaia/PA

\title{
Performance of bioclimatic equations for estimation of reference evapotranspiration in Conceição do Araguaia/PA
}

\begin{abstract}
:
Due to uncertainties in rainfall totals that affect a region because of climate change, agriculture needs to have techniques that minimize the inherent risks of this activity. ETo originally introduced under the term evapotranspiration potential (ETp), is an important agrometeorological parameter, mainly for irrigation planning and management. There are several equations for calculating ETo, from the simplest ones, on a purely empirical basis, to those that have a consistent physical foundation on the phenomenon, however, there is always some empiricism present. Therefore, the objective of this study was to evaluate the performance of four methods (Turc, Hargreaves and Samani, Jesen-Haise and Makkink) for estimating daily ETo by means of comparisons with the standard Penman-Monteith-FAO method56, in Conceição of Araguaia/PA. The meteorological data were taken from the National Institute of Meteorology in the period of 26 years (1990-2016). The standard method was Penman-Monteith-FAO56, and the comparison of results was by means of the determination coefficient $\left(R^{2}\right)$, WIllmott's concordance index (d), Pearson's correlation coefficient ( $\mathrm{r}$ ) and coefficient of confidence (c). It was observed that in the climatic conditions of Conceição do Araguaia / PA, the Turc and Hargreavis and Samani methods were the ones that best estimated the reference evapotranspiration (ETo) when compared to the Penman-Monteith-FAO standard. Key words: Agrometeorology, evaporation, irrigation, Penman-Monteith.
\end{abstract}

\section{Rendimiento de ecuaciones bioclimáticas para estimación de la evapotranspiración de referencia en Conceição do Araguaia / PA}

\section{Resumen:}

Debido a las incertidumbres en los totales pluviométricos que inciden sobre una región a causa del cambio climático, la agricultura necesita disponer de técnicas que minimicen los riesgos inherentes a esa actividad. La ETo originalmente introducida bajo el término evapotranspiración potencial (ETP), es un importante parámetro agrometeorológico, principalmente para la planificación y manejo de riego. Hay varias ecuaciones para calcular la ETo, desde los más simples, con base puramente empírica, hasta aquellas que presentan basamento físico consistente sobre el fenómeno, sin embargo, siempre hay algún empirismo presente. En el presente trabajo se evaluó el desempeño de cuatro métodos (Turc, Hargreaves y Samani, Jesen-Haise y Makkink) para la estimación de la ETo diaria a través de comparaciones con el método estándar de Penman-Monteith-FA056, en Conceição del Araguaia / PA. Los datos meteorológicos fueron retirados del Instituto Nacional de Meteorología en el período de 26 años (1990-2016). El método adoptado por defecto fue el de Penman-Monteith-FA056, y la comparación de los resultados fue por el coeficiente de determinación $\left(\mathrm{R}^{2}\right)$, índice de concordancia de Willmott $(\mathrm{d})$, coeficiente de correlación de Pearson ( $\mathrm{r}$ ) y del coeficiente de correlación de Pearson ( $\mathrm{r}$ ) de confianza (c). Se observó que en las condiciones climáticas de Concepción del Araguaia / PA, los métodos de Turc y Hargreavis y Samani fueron los que mejor estimaron la evapotranspiración de referencia (ETo) en comparación con el patrón Penman-Monteith-FAO.

Palabras clave: Agrometeorología, evaporación, irrigación, Penman-Monteith.

\section{INTRODUÇÃO}

A escassez de água se transformou nos últimos anos em um dos temas mais discutidos, entre os setores que mais consomem esse recurso está a agricultura irrigada que 
sozinha é responsável por cerca de $72 \%$ da água doce total consumida. No Brasil este consumo é de cerca de 63\% (PEREIRA, 2006).

A evapotranspiração é uma variável fundamental para obter a necessidade hídrica das culturas, e também fornece subsídio para o dimensionamento de sistemas de bombeamento, adução e distribuição de água na irrigação (ABABAEI, 2014; FERRAZ et al., 2014, MANCOSU et al., 2014, CAPORUSSO e ROLIM, 2015). Os métodos empregados na quantificação da ETo podem ser diretos ou indiretos. Os métodos considerados indiretos baseiam-se em equações parametrizadas que empregam dados meteorológicos, muitas vezes não disponíveis na propriedade ou na cidade de interesse (SOUZA et al., (2015).

Diante disso, para se obter economia dos recursos hídricos utilizados na agricultura irrigada, é de fundamental importância dispor de sistemas de irrigação bem planejados que levem em consideração tanto as características da planta como também as características meteorológicas da região, principalmente da evapotranspiração de referência ETo (MENDONÇA; DANTAS, 2010). Embora seja considerado o modelo mais eficiente para obter a ETo, a equação padrão da FAO depende de muitas variáveis meteorológicas para seu cálculo, que nem sempre estão disponíveis nos locais de avaliação (PAZ; THEBALDI, 2018,).

Devido às restrições de uso da equação padrão, em função da quantidade de variáveis meteorológicas, outras equações têm sido utilizadas nas equações mais simples, que exigem uma quantidade menor de variáveis meteorológicas, alguns deles apresentam resultados satisfatórios e têm boa aplicabilidade (DA PAZ; THEBALDI, 2018).

No entanto, antes de escolher um método de estimativa de ETo para uma determinada região, é necessário verificar o desempenho deste, vários estudos estão sendo realizados por pesquisadores que compararam os mais diferentes métodos presentes na literatura, com a intenção de encontrar métodos representativos para condições locais específicas (KISI, 2014).

Baseado nessas informações, objetivou-se com esse trabalho avaliar o desempenho de quatro métodos (Turc, Hargreaves e Samani, Jesen-Haise e Makkink) para estimativa da ETo diária por meio de comparações com o método padrão de Penman-Monteith-FAO56, em Conceição do Araguaia/PA. 


\section{MATERIAL E MÉTODOS}

O trabalho foi realizado a partir dos dados diários de temperatura máxima e mínima do ar, umidade relativa do ar, e velocidade do vento, provenientes da Estação Convencional

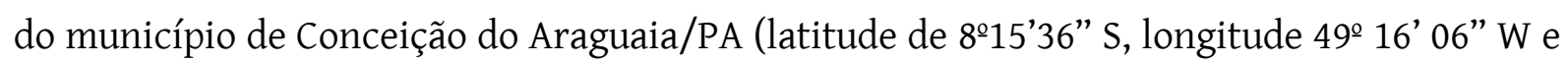
altitude 156,85 m), compreendendo o período de 1990 a 2016. Os dados foram obtidos do Banco de Dados Meteorológicos para Ensino e Pesquisa (BDMEP) disponibilizados pelo Instituto Nacional de Meteorologia (INMET).

A área de estudo possui clima equatorial super-úmido, com temperatura média anual de $26,3^{\circ} \mathrm{C}$, período chuvoso de novembro a maio e precipitação pluvial total anual de 2000 $\mathrm{mm}$ (SILVA, 2015).

Os métodos utilizados para a estimativa da ETo foram: Hargreaves e Samani, e Makkink, Turc, Jensen-Haise. Todos os métodos foram comparados com o método de Penman-Monteith, parametrizado pela FAO56 no seu manual (ALLEN et al., 1998). As metodologias utilizadas na presente pesquisa estão apresentadas na tabela 1.

Tabela 1- Metodologias utilizadas no estudo.

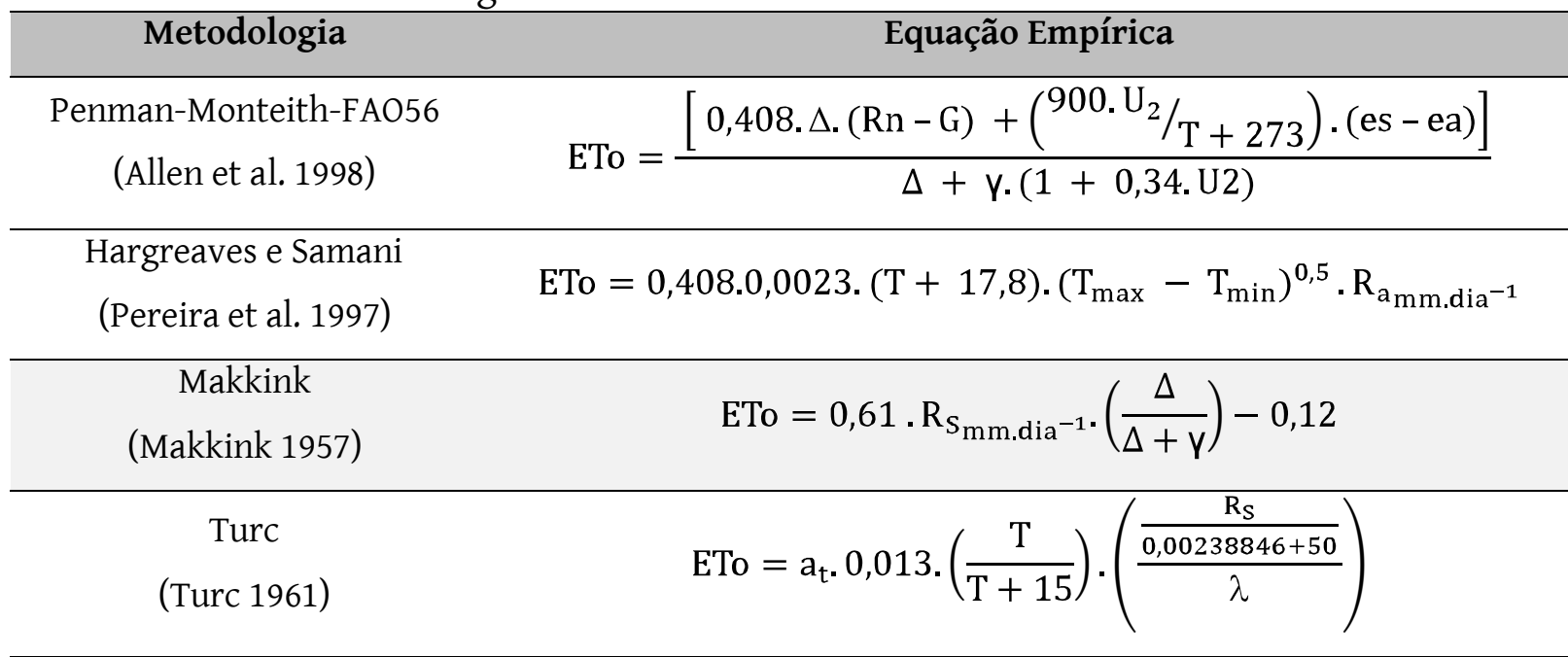

Jensen e Haise

(Jensen e Haise 1963)

$$
\mathrm{ETo}=\mathrm{R}_{\mathrm{Smm.dia}^{-1}}(0,025 . \mathrm{T}+0,078)
$$

Legenda: $\Delta=$ declividade da curva de pressão $\left(\mathrm{kPa}^{\circ} \mathrm{C}^{-1}\right) ; \mathrm{Rn}=$ saldo de radiação $\left(\mathrm{MJ} \mathrm{m}^{-2}\right.$ dia $\left.^{-1}\right) ; \mathrm{G}=$ fluxo de calor $\left(\mathrm{MJ} \mathrm{m}^{-2}\right.$ dia $\left.{ }^{-1}\right) ; \gamma=$ constante psicrométrica $\left(\mathrm{MJ} \mathrm{kg}^{-1}\right) ; \mathrm{T}=$ temperatura média $\left({ }^{\circ} \mathrm{C}\right) ; \mathrm{U}_{2}=$ velocidade média do vento $\left(\mathrm{m} \mathrm{s}^{-1}\right)$; es = pressão de saturação de vapor $(\mathrm{kPa})$; ea = pressão de vapor $(\mathrm{kPa}) ; \mathrm{Tmax}=$ temperatura máxima $\left({ }^{\circ} \mathrm{C}\right) ; \mathrm{Tmin}=$ temperatura mínima $\left({ }^{\circ} \mathrm{C}\right) ; \mathrm{Ra}_{\mathrm{mm} \text {.dia }}{ }^{-1}=$ Radiação extraterrestre $\left(\mathrm{mm} \cdot \mathrm{dia}^{-1}\right) ; \mathrm{Rs}_{\mathrm{mm} . \text { dia }}{ }^{-1}=$ radiação global $\left(\mathrm{mm} \cdot \mathrm{dia}^{-1}\right) ; \lambda=$ calor latente de vaporização $\left(\mathrm{MJ} . \mathrm{mm}^{-1}\right) ; \mathrm{a}_{\mathrm{t}}$ fator da umidade relativa (\%).

Elaborado pelo autor 
Na comparação dos valores de ETo obtidos pelos métodos empíricos em comparação ao padrão Penman-Monteith-FA056 foi considerado a análise de correlação e regressão linear para obtenção dos coeficientes da equação $(Y=a+b x)$. Para a avaliação do desempenho estatístico dos modelos, foram empregados os indicadores de coeficiente de correlação $(r)$, coeficiente de determinação $\left(R^{2}\right)$, índice de concordância (d) de Willmott et al. (1985) e o índice de confiança ou desempenho (c). Sendo expresso pelas equações 2, 3 e 1 respectivamente

$$
\begin{aligned}
& \mathrm{d}=1-\left[\frac{\sum(\mathrm{Pi}-\mathrm{Oi})^{2}}{\sum(|\mathrm{Pi}-\mathrm{O}|+|\mathrm{Oi}-\mathrm{O}|)^{2}}\right] \\
& \mathrm{c}=\mathrm{r} \cdot \mathrm{d} \\
& \mathrm{r}=\frac{\sum_{\mathrm{i}=1}^{\mathrm{N}}(\mathrm{Oi}-\mathrm{O}) \cdot(\mathrm{Pi}-\mathrm{P})}{\sqrt{\sum_{\mathrm{i}=1}^{\mathrm{N}}(\mathrm{Oi}-\mathrm{O})^{2}} \cdot \sqrt{\sum_{\mathrm{i}=1}^{\mathrm{N}}(\mathrm{Pi}-\mathrm{P})^{2}}}
\end{aligned}
$$

Onde, $\mathrm{r}$ = coeficiente de correlação de Pearson; $\mathrm{d}$ = coeficiente de concordância; $\mathrm{c}=$ índice de confiança; $\mathrm{Pi}=$ evapotranspiração estimada pelo método testado (mm.d-1); P a média da evapotranspiração do método testado (mm.d-1 ${ }^{1}$; Oi = evapotranspiração estimada pelo método padrão $\left(\mathrm{mm}^{\mathrm{d}-\mathrm{l}}{ }^{\mathrm{l}}\right.$; e $\mathrm{O}=$ média dos valores observados pelo método padrão (mm.d-1).

O coeficiente c, proposto por Camargo e Sentelhas (1997), é interpretado de acordo com os referidos autores como: "ótimo" (c > 0,85); "muito bom" $(0,76<c<0,85)$; "bom" $(0,66$ $<$ c < 0,75); "mediano" $(0,61<\mathrm{c}<0,65)$, "sofrível" $(0,51<\mathrm{c}<0,60)$, "mau" $(0,41<\mathrm{c}<0,50)$ e "péssimo" $(c<0,40)$.

\section{RESULTADOS E DISCUSSÃO}

Na tabela 2, estão apresentados o coeficiente de determinação $\left(\mathrm{R}^{2}\right)$, índice de Concordância (d), Coeficiente de correlação de Pearson (r) e índice de confiança (c) da correlação dos modelos empíricos com o método padrão, Penman Monteith para o período analisado (1990-2016) na cidade de Conceição do Araguaia/PA. 
Tabela 2 - Desempenho dos métodos de estimativa da ETo diário, entre os anos de 1990 a 2016 em Conceição do Araguaia/PA.

\begin{tabular}{cccccc}
\hline Métodos & $\mathbf{R}^{\mathbf{2}}$ & $\mathbf{R}$ & $\mathbf{D}$ & $\mathbf{c}$ & Desempenho \\
\hline Turc & 0,70081 & 0,83715 & 0,82 & 0,69 & Bom \\
Hargreaves e Samani & 0,75027 & 0,86618 & 0,77 & 0,67 & Bom \\
Jensen Haise & 0,77373 & 0,87962 & 0,62 & 0,55 & Sofrível \\
Makkink & 0,71610 & 0,84623 & 0,69 & 0,58 & Sofrível \\
\hline
\end{tabular}

Elaborado pelo autor.

O método que apresentou melhor confiabilidade foi Turc, apresentando um desempenho bom de acordo com o critério proposto por Camargo e Sentelhas (1997), com o valor de $\mathrm{c}=0,69$, seguido pelo método de Hargreaves e Samani que também foi classificado como bom com um valor de $\mathrm{c}=0,67$. Por outro lado, os métodos de Jensen Haise e Makkink foram os que mostraram menor confiabilidade, apresentando um desempenho sofrível, com o valor de $\mathrm{c}=0,55$ e 0,58 respectivamente.

Os resultados aqui encontrados se assemelham aos encontrados por Fanaya Júnior et al., (2012) que avaliando métodos de estimativa da evapotranspiração de referência em Aquidauana/MS também obtiveram desempenho bom para o método de Turc. 0 bom desempenho desse método pode ser explicado pelo fato do mesmo ser baseado na radiação, e geralmente os métodos baseados na radiação apresentam melhor desempenho quando comparados com o método padrão, entretanto, vale ressaltar que essa não é uma regra, visto que a evapotranspiração é afetada por vários fatores.

Observa-se neste trabalho que o método de Hargreaves e Samani não é tão preciso quanto o método de Turc quando comparados com o método padrão, no entanto, percebe-se que este método apresentou um bom desempenho com um índice de concordância $d=0,77$, coeficiente de correlação $r=0,86818$ e coeficiente de determinação $R^{2}=0,75027$ (Figura 1B) podendo assim na ausência do método de Turc ser usado para estimativa da ETo em Conceição do Araguaia/PA. Esses resultados diferem dos encontrados por Passos et al., (2017) que avaliando métodos de estimativa da ETo em Chapadinha/MA obtiveram classificação de péssimo por esse método.

De acordo com os resultados obtidos no período analisado, percebe-se que a maior inclinação das retas dos modelos lineares que demonstram a correlação do método padrão 
com os empíricos foi encontrado pelo método de Jesen Haise com um $\mathrm{R}^{2}=0,7737$ (Figura 1C). Embora esses valores sejam altos, não predizem um bom resultado do conjunto do método, que deve ser interpretado conforme o índice de concordância (d), que expressa uma medida de exatidão do método; o coeficiente de correlação ( $r$, que expressa uma medida da precisão do método; e o índice "c", que expressa um desempenho conjunto do método (CAMARGO; SENTELHAS, 1997). O desempenho sofrível encontrado aqui por esse método, pode ser explicado pelo fato de que de acordo com Subburayan et al., (2011) o mesmo é um método indicado para regiões de clima árido e semiárido, diferentes do clima da região aqui estudada.

Figura 1 - Regressão linear entre os valores diários de evapotranspiração de referência (ETo) estimados pela comparação dos métodos de Turc (A), HargreavesSamani (B), Jensen-Haise (C) e Makkink (D) com o método padrão FAO PenmanMonteith, para o município de Conceição do Araguaia/PA.
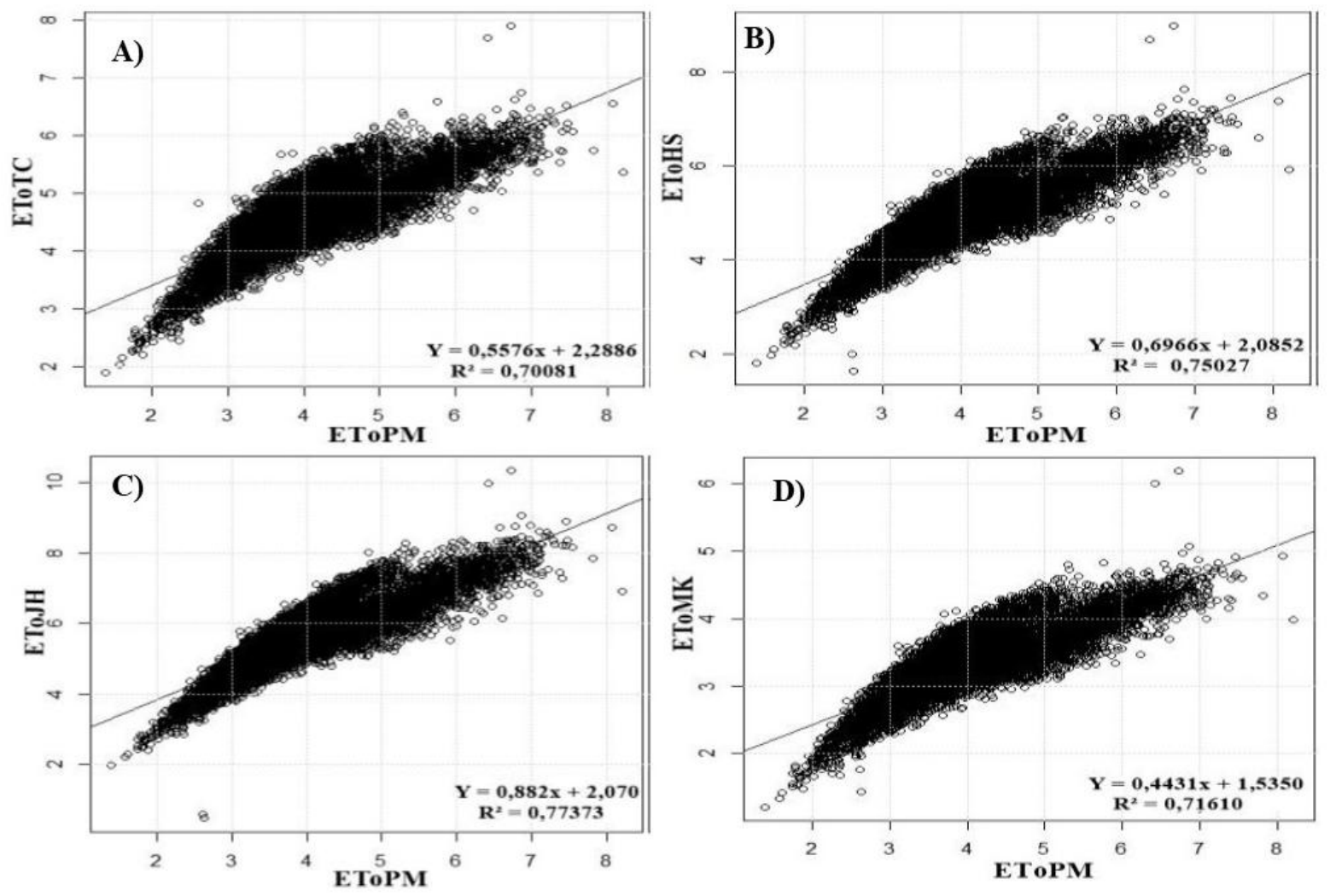

Fonte: Os Autores, 2019.

A equação de Jensen e Haise foi uma equação desenvolvida para regiões áridas e semi-áridas, o que explica sua boa adaptabilidade para o período seco, ao contrário do período úmido, condições em que este trabalho foi executado, o que acarretou um baixo desempenho desse método. 
O método de Makkink embora tenha apresentado um valor satisfatório para o coeficiente de determinação $R^{2}=0,71610$ (Figura 1D), foi classificado como sofrível apresentando um coeficiente de confiança $\mathrm{c}=0,58$, esse desempenho pode está relacionado ao fato de que o método de Makkink foi desenvolvido para regiões frias diferentes das condições onde o trabalho foi executado. Resultados diferentes aos aqui encontrados foram observados por Ferronato et al., (2016), que testando métodos de estimativa da ETo em Santo Antônio do Leverger/MT obtiveram ótimo desempenho por esse método.

Os métodos Makkink e Jensen-haise se caracterizam pela simplicidade de suas equações e pelo número reduzido de parâmetros de entrada. Apesar dessas vantagens, esses métodos citados não devem ser utilizados para estimativa da ETo para a região do município de Conceição do Araguaia/PA.

\section{CONCLUSÕES}

Nas condições climáticas de Conceição do Araguaia/PA, os métodos de Turc e Hargreavis e Samani foram os que melhor estimaram a evapotranspiração de referência (ETo) quando comparado com o padrão Penman-Monteith-FAO. Em substituição ao método padrão na estimativa da ETo local, recomenda-se o método Turc.

\section{REFERÊNCIAS}

ABABAEI, B. Are weather generators robust tools to study daily reference evapotranspiration and irrigation requirement. Water Resource Management, v. 28(1): 915-932, 2014. Disponível em: < https://link.springer.com/article/10.1007/s11269-014-0524-3>. doi: 10.1007 / s11269-014-0524-3. Acesso em: $11 \mathrm{abr} .2019$.

ALLEN, R. G.; PEREIRA, L. S.; RAES, D.; SMUTH, M. Crop Evapotranpiration: Guidelines for computing crop water requirements, Rome: FAO, 1998, 301p. Disponível em: < http://www.fao.org/3/X0490E/X0490E00.htm>.Acesso em: 11 abr. 2019.

CAMARGO, A. P.; SENTELHAS, P.C. Avaliação do desempenho de diferentes métodos de estimativa da evapotranspiração potencial no Estado de São Paulo, Brasil. Rev. Bras. Agrometeorologia. Santa Maria, v.5, n.1, p.89-97, $1997 . \quad$ Disponível em: https://www.researchgate.net/publication/284194031_Avaliacao_do_desempenho_de_diferentes_metodos_d e_estimativa_da_evapotranspiracao_potencial_no_Estado_de_Sao_Paulo_Brasil>. Acesso em: 12 abr. 2019. 
CAPORUSSO, N. B.; ROLIM, G. de S. Reference evapotranspiration models using different time scales in the Jaboticabal region of São Paulo, Brazil. Acta Scientiarum. Agronomy (Impresso), v. 37, p. 1-9, 2015. Disponível em: < http://dx.doi.org/10.4025/actasciagron.v37i1.18277

>. doi: 10.4025/actasciagron.v37i1.18277. Acesso em: 10 abr. 2019.

FERRAZ, R. C.; Estimativa da evapotranspiração de referência utilizando redes neurais artificiais para o Estado do Rio Grande do Sul. Revista Tecnológica v.23, p.25-31, 2014. Disponível em: < http://dx.doi.org/10.4025/revtecnol.v23i1.20908.g14567

>. doi:10.4025/revtecnol.v23i1.20908.g14567. Acesso em: 21 abr. 2019.

FERRONATO, A.; CHIG, L. A.; GOULART, D. B.; JÚNIO, J. H. C.; PEREIRA, L. C.; BIUDES, M. S. Métodos de estimativa da evapotranspiração de referência para Santo Antônio do Leverger-MT. Revista de Ciências Agroambiental. v.14, n.1, p.110-118, 2016. Disponível em: < https://periodicos.unemat.br/index.php/rcaa/article/view/1418

>. Acesso em: 09 abr. 2019.

FANAYA JÚNIOR, E. D.; LOPES, A. D.; OLIVEIRA, G. Q.; JUNG, L. H. Métodos empíricos para estimativa da evapotranspiração de referência para Aquidauana, MS. Irriga, Botucatu, v. 17, n. 4, p. 418 - 434, outubro dezembro, 2012. Disponível em: < http://revistas.fca.unesp.br/index.php/irriga/article/view/282>. Acesso em: 11 mai. 2019.

JENSEN, M. E. HAISE, H. R. Estimating evapotranspiration from solar radiation. Journal of the Irrigation and Drainage Division-ASCE, v.4, n. 1, p. 15-41, 1963.

KISI, O. Comparison of different empirical methods for estimating daily reference evapotranspiration in Mediterranean climate. Journal of Irrigation and Drainage Engineering, New York, v.140, n.1, p.1-7, 2014. Disponível em: <https://doi.org/10.1061/(ASCE)IR.1943-4774.0000664>. doi: 10.1061/(ASCE)IR.19434774.0000664. 11 mai. 2019.

MAKKINK, G. F. Ekzamento de la formulo de Penman. Netherlands Journal of Agricultural Science, Wageningen, v.5, n.2, p290-305, 1957.

MANCOSU, N.; SNYDER, R. L.; SPANO, D. Procedures to develop a standardized reference evapotranspiration zone map. Journal of Irrigation and Drainage Engineering, New York, v.140, n.9, p.1-11, 2014. Disponível em: < https://doi.org/10.1061/(ASCE)IR.1943-4774.0000697

>. doi: 10.1061/(ASCE)IR.1943-4774.0000697. Acesso em: 11 mai. 2019.

MENDONÇA, E. A.; DANTAS, R. T. Estimativa da evapotranspiração de referência no município de Capim, PB. Revista Brasileira de Engenharia Agrícola e Ambiental, Campina Grande, v. 14, n. 2, p. 196-202, 2010. Disponível em: < http://www.scielo.br/scielo.php?pid=S1415-43662010000200011\&script=sci_abstract\&tlng=E S>. Acesso em: 11 jun. 2019.

PASSOS, M. L. V.; RAPOSO, A. B.; MENDES, T. J. Evapotranspiração de referência por diferentes métodos para o município de Chapadinha-MA. Brazilian Journal of Applied Technology for Agricultural Science, Guarapuava-PR, v.10, n.1, p.59-66, 2017. Disponível em: < http://revistas.ufcg.edu.br/acsa/index.php/ACSA/article/view/868

>. Acesso em: 11 jun. 2019.

PEREIRA, A. R., VILLA NOVA, N. A., SEDIYAMA, G. C. Evapotranspiração. 1.ed. Piracicaba: FEALQ, 183p. 1997.

PEREIRA, J. B. A. Avaliação do crescimento, necessidade hídrica e eficiência no uso da água pela cultura do pimentão (capsicum annuum. L), sob manejo orgânico nos sistemas de plantio com preparo de solo e direto - Seropédica - RJ. 2006. 45f. Dissertação (Mestrado em Fitotecnia) - Universidade Federal Rural do Rio de Janeiro, Seropédica/RJ. Disponível em: <http://www.ufrrj.br/institutos/it/deng/daniel/Downloads/Material/Teses\%200rientadas/TESE\%20Joao\%20 Batista.pdf $>$. Acesso em: 10 mai. 2019.

SILVA, J.L. C. Perfil do produtor de abacaxi e incidência da fusariose em Conceição do Araguaia, PA. 2015. 47f. Dissertação (Mestrado profissional em Defesa Sanitária Vegetal) - Universidade Federal de Viçosa, 
Desempenho de equações bioclimáticas para estimativa da evapotranspiração de referência em Conceição do Araguaia/PA

Viçosa/MG.

Disponível

em:

http://www.locus.ufv.br/bitstream/handle/123456789/9529/texto\%20completo.pdf?sequence=1\&isAllowed=y

>. Acesso em: 01 mai. 2019.

SOUZA, A. P., DE ALMEIDA, F. T., ARANTES, K. R., MARTIM, C. C., SILVA, J. O. Coeficientes de Tanque Classe A para estimativa da evapotranspiração de referência diária na região de transição Cerrado-Amazônica. Scientia Plena, v. 11, n. 5, 2015. Disponível em: < https://www.scientiaplena.org.br/sp/article/view/2383/1216

>. Acesso em: 12 mai. 2019.

SUBBURAYAN, S.; MURUGAPPAN, A., MOHAN, S. Modified Hargreaves Equation for Estimation of ETo in a Hot and Humid Location in Tamilnadu State, India. International Journal of Engineering Science and Technology, Tehran, v. 3, n $1, \quad 2011$. Disponível em: < https://www.researchgate.net/publication/50392177_Modified_Hargreaves_Equation_for_Estimation_of_ET0 in_a_Hot_and_Humid_Location_in_Tamilnadu_State_India

>. Acesso em: 18 mai. 2019.

TURC, L. Évaluation des besoins en eau d'irrigation, évapotranspiration potentielle. Annales Agronomiques, v. 12 , n. 1 ,p.13-49. 1961.

WILLMOT, C. J.; ACKLESON, S. G.; DAVIS, J. J.; FEDDEMA, K.; KLINK, D. R. Statistics for the evaluation and comparison of models. Journal of Geophysical Research, Ottawa, v. 90, n. 5, p. 8995-9005, 1985. Disponível $\mathrm{em}:<\underline{\text { http://climate.geog.udel.edu/ climate/publication_html/Pdf/WADFKLO_JGROceans_85.pdf }}$ >. Acesso em: 11 mai. 2019.

\section{(cc) $\mathrm{EY}$}

Este trabalho está licenciado com uma Licença Creative Commons - Atribuição 4.0 Internacional. 